\title{
Detection of Landing Areas for Unmanned Aerial Vehicles
}

\author{
Kausar Mukadam \\ Student, Dwarkadas J. \\ Sanghvi \\ College of Engineering \\ Plot No.U-15, J.V.P.D. \\ Scheme, \\ Bhaktivedanta Swami \\ Marg, \\ Vile Parle (West), \\ Mumbai-400 056. \\ India
}

\author{
Fuzail Misarwala \\ Student, Dwarkadas J. \\ Sanghvi \\ College of Engineering \\ Plot No.U-15, J.V.P.D. \\ Scheme, \\ Bhaktivedanta Swami \\ Marg, \\ Vile Parle (West), \\ Mumbai-400 056. \\ India
}

\author{
Aishwarya Sinh \\ Student, Dwarkadas J. \\ Sanghvi \\ College of Engineering \\ Plot No.U-15, J.V.P.D. \\ Scheme, \\ Bhaktivedanta Swami \\ Marg, \\ Vile Parle (West), \\ Mumbai-400 056. \\ India
}

\author{
Ruhina Karani \\ Asst. Professor, \\ Dwarkadas J. Sanghvi \\ College of Engineering \\ Plot No.U-15, J.V.P.D. \\ Scheme, \\ Bhaktivedanta Swami \\ Marg, \\ Vile Parle (West), \\ Mumbai-400 056. \\ India
}

\begin{abstract}
Unmanned aerial vehicles (UAV), commonly called drones, are a growing field in computer technology with applications ranging from military to delivery systems. One of the foremost obstructions to the allowance of UAV journeys over populated areas or civilian airspace is the lack of sophisticated automated systems that detect UAV landing sites. In this paper, we propose a landing area detection system, based primarily on machine learning that focuses on determining drop-off points. Determining a prime drop site within a property is an important aspect of automated delivery systems. Our proposed method uses features such as the colour and texture of pixels to describe the characteristics of an area. These characteristics are employed by machine learning algorithms such as Support Vector Machine, to predict appropriate drop-off locations.
\end{abstract}

\section{Keywords}

Landing Area Detection; UAV; Unmanned Aerial Vehicles.

\section{INTRODUCTION}

One of the major research areas in the field of robotics, in the last decade, has been the development of UAV's which can be used for a wide range of applications. An unmanned aerial vehicle (UAV) is primarily an aircraft without a human pilot aboard, whose flight may be controlled either autonomously through on-board computers or remotely by a pilot on ground. An unmanned vehicle that operates autonomously or is remotely piloted eliminates risks, such as pilot safety, and increase the overall effectiveness [1].

Delivery systems have emerged as one of the most talked about applications of these UAV's. Amazon has promised a futuristic delivery system, Amazon Prime Air, which will deliver packages to their customers within 30 minutes or less by using UAV's. A key component in a successful delivery is the detection of a safe landing area for the UAV or parcel at the delivery location. For example, dropping a package on a doorstep or driveway is preferable to a delivery on the roof or into a tree.

In this paper, we have proposed an algorithm that uses satellite images obtained through a delivery address to detect safe landing sites. These images are processed and analysed to extract features and characteristics that can aid in this determination. The images are retrieved through Google Static Maps, and features such as colour and texture are extracted.
These features form the basis for machine learning algorithms like Support Vector Machine, which then identify a suitable landing area from the image.

\section{LITERATURE SURVEY}

Substantial research has been conducted in the field of aerial imagery and its analysis ( [2]), which can be applied in the detection of safe landing areas. Further many researchers ( [1] [3] [4]) have also researched various methods to identify landing areas.

One such method has been proposed by Josh Beal and Gabe Alvarez [3], which targets delivery systems using UAV's, which is depicted in Figure 1. The purpose of their research was to determine the optimal drop off location within a property for an autonomous delivery system using an unmanned air vehicle. The training data used was gathered using pre-determined addresses from a service Maponics, which were then applied to the Google Static Maps API for obtaining images of those addresses.

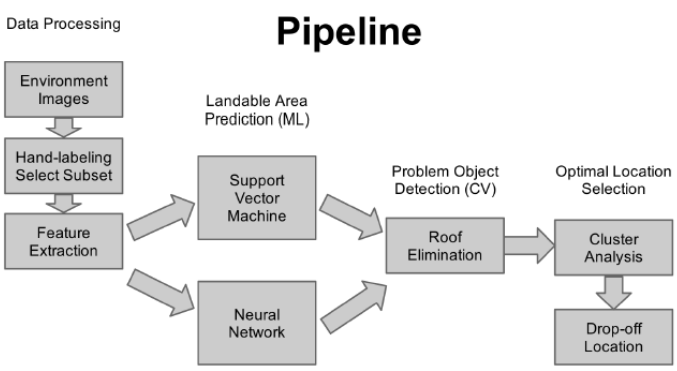

Figure 1. Pipeline and future directions [5]

Colour, using the RGB Model, and texture, using Canny Edge Detection Algorithm, formed the feature set in this paper. Initially, single pixels were used as training examples but due to the inaccuracy of the results in this method, and the excessive convergence time taken for SVM algorithm, the methodology was re-worked and small square patches were used instead. A threshold for classifying a patch as land-able was set at $45 \%$, i.e. a patch will be classified as land-able if $45 \%$ or more of its pixels were land-able.

For the training, SVM algorithm was used through the libsvm package, by using scripts that converted images into feature vectors and vice-versa. The specific parameters for the 
algorithms, for example the Gaussian Kernel approach, were improved multiple times over the course of the project.

Learning was also implemented using Neural Networks, due to their previous use in aerial imagery detection. Feed Forward Neural Networks were implemented using MATLAB with little success as very few land-able areas were detected. An overall cross-validation test error of $9.5 \%$ was recorded in the experiments.

Future work included the use of roof detection algorithms to eliminate patches containing roofs, so as to increase the efficiency of the delivery system.

Research by Xufeng Guo et. al [4] aims at detecting the safest landing site for an UAV in the event of an emergency like a hardware or software malfunction. Here a safe landing area is an uninhabited area or an area free from critical infrastructure. Various factors that present barriers to this detection include inconsistent image resolution due to altitude changes and environmental changes. Using Gaussian Mixture Model (GMM) and Support Vector Machines (SVM) coupled with a footprint operator for feature extraction, the presented system's performance was compared with a baseline Artificial Neural Network system and improved results have been obtained.

The images taken by the UAV camera are sampled into square windows of a predefined number of pixels and a certain overlapping ratio. This is followed by feature extraction (colour and texture) of each extracted segment. Colour features are obtained using the HSV (Hue, Saturation, Value) model whereas the texture features are analysed using HOG (Histogram of Oriented Gradients) and LBP(Local Binary Patterns). Feature extraction has been done with the help of a footprint mask generated by the spoke wheel operator. This helps in eliminating regions having clearly different characteristics within a patch enabling better representation of the dominant area. The flatness of the area around a given pixel was also extracted. Another important feature obtained was the shape of the area neighbouring a given pixel. This information is arrived at by using the length of the spoke wheel of the footprint.

The classifiers chosen were GMM and SVM which are trained for each individual feature and order. In GMM, five different Gaussian distributions were taken that exponentially increase by a factor of 2. SVM training was done using three SVM kernels - linear, Radial Basis Function and polynomial.

The datasets used for evaluation included all terrains covering urban, suburban, and rural and forest areas. The data collection contained images at three different resolutions.

The minimum area required for landing the target UAV was varied in accordance to the zoom levels. Every image was manually labelled as 'safe' for landing (logical 1) or 'unsafe' (logical 0). In training, different resolution images were used to produce classifiers adaptive to all zoom levels. However in testing, different resolution images were tested separately in order to note the classifier's performance at varying zoom levels.

The performance of the system was evaluated based on evaluation metrics of correctness, completeness and quality factor. Completeness represents the percentage of all correctly detected landing areas marked 'safe'. Correctness, on the other hand, is the percentage of all correctly detected landing areas labelled 'unsafe'. The quality factor is a measure of the correctly detected 'safe' area to the entire detected and all available 'safe' area.

The experimental results obtained showed that the polynomial SVM classifier gave better results over a wide range of parameters. As far as the individual features are concerned, Spoke length and HOG provide best results while LBP and footprint perform poorly. All evaluation metrics (completeness, correctness and quality factor) increased through the use of this system and an improvement of approximately thirty-six percent was obtained in the overall score, making the proposed system more stable and immune as compared to the baseline system.

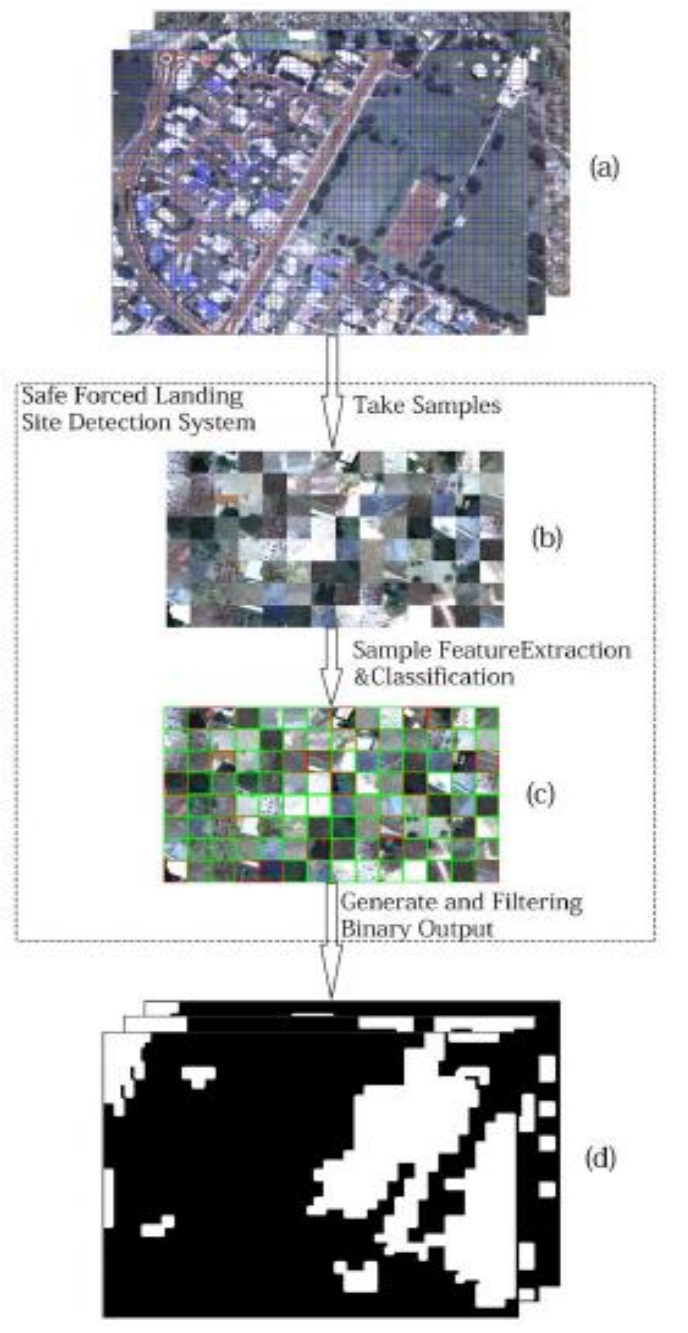

Figure 2. System Overview [4] where original image (a) is sampled (b). Feature extraction and classification is performed for each segment (c). Classification result is reflected in a binary image, and the filtered binary image is the output $(d)$.

Seyfallah Bouraoui et. al. [2] have proposed an approach for identifying residential areas, trees, forest land, houses and streets using region adjacency graphs. Nodes of the graph represent shape like houses or trees, and arcs refer to the adjacency relation amid them.

The data set consists of multi-spectral satellite images with high resolution. In pre-processing, the structures of interest were illuminated and the image was segmented. 
The multispectral segmentation has been done using the normalized difference Water index (NDWI) to deduce cultural activity and water bodies. Region growing segmentations is used to extract houses and streets. An RGB image is used and histogram equalization is applied on the image colours. Image segmentation is done by growing each region around a starting pixel based on a threshold value.

For trees and forests detection, vegetation indices are used that estimate the vegetation density from satellite and airborne images. NDVI is based on the reflective values in near infrared and red bands [5].

For identifying houses, roof detection is used. Classification is carried out to identify the red roofs colour, and the boundaries of the roofs are detected. Mathematical morphology is used to extract streets based on their shape. Lines are extracted by applying erosion and dilatation operations of mathematical morphology based on four structuring elements- horizontal, vertical and two diagonals.

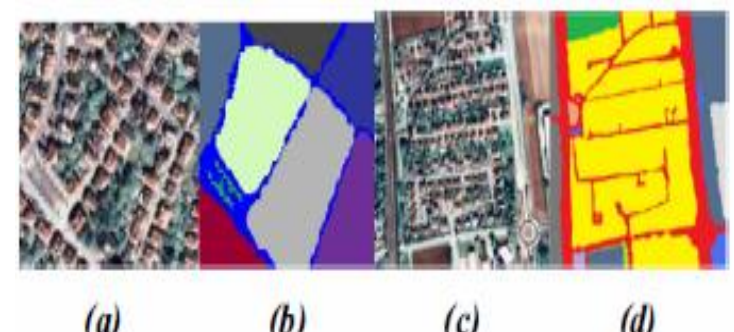

Figure 3. Result [4] (a) original image, (b) result (c) image containing non-residential areas (d) result: residential areas in yellow.

To detect residential areas, all the structures detected previously are gathered on the same image and other small regions that aren't a structure are removed. This is done using the region growing algorithm. In the end, the number of residential areas in the segmented image are counted by graph formalization. Automaton language and regular expressions are used to determine relationships between nodes (structures) in the graph and hence recognize residential areas.

\section{PROPOSED METHOD}

The system proposed in this paper uses feature extraction and machine learning algorithms to identify suitable landing areas. The system description is given in Figure 4. The following sections detail each step of the process for detection of landing areas.

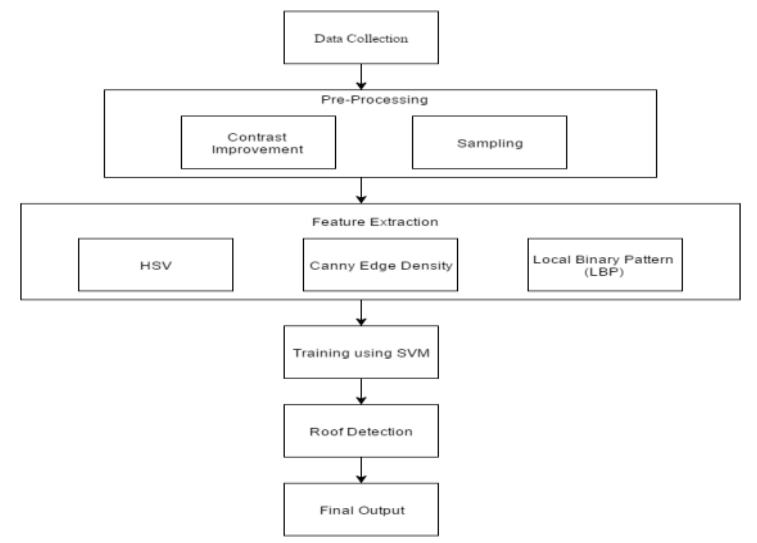

Figure 4. Diagrammatic representation of proposed method.

\subsection{Pre-processing}

The input for this step is a satellite image of the delivery location or address for UAV. This data set has been constructed by using satellite images from Google Earth Pro, which have been extracted on the basis of delivery addresses. This data set covers a varied terrain that the UAV may encounter. First, the contrast of the images is enhanced. The images are then sampled into small square patches with an edge of size 25 pixels and an overlapping ratio of 50\%. The system implements feature extraction on each extracted segment, using both colour and texture features.

\subsection{Feature Extraction}

For our system, the chosen features include colour and texture features. In the past colour features have been extracted using either the RGB colour model [3] or HSV colour model [4]

In the RGB colour model, the images are represented by features including average red, green, and blue values, while in the HSV model, Hue, Saturation and Value (brightness) is calculated. The HSV model is motivated by the human visual system, whereas the RGB representation is not very suited for describing image colour from a human point of view [6]. Further, experiments conducted by Michael W. Schwarz et. al. [7] have shown that while RGB colour model is quicker, the results are inaccurate as compared to the HSV colour model.

Therefore in our system the HSV Feature is used to represent colour feature, but for the purpose of performance comparison, features will also be extracted in the RGB colour space. The image segments are converted from an RGB colour model to the HSV colour model. Then the average hue, saturation and intensity values of the patch pixels will be calculated which forms a three dimensional HSV feature.

Texture, an inherent property of almost all surfaces, is one of the significant characteristics in identifying objects or regions of interest in images. It can be assessed as being fine, coarse, smooth, irregular, or lineated. [8].

Texture features are computed using edge density and Local binary patterns (LBP). Edge density is derived through the Canny edge detection algorithm. The edge density feature of each patch is calculated by calculating the number of pixels in each patch that are edges. Further texture features for a patch are computed by using the edge densities of areas surrounding the patch. The concept is that areas of an image corresponding to a structure like a tree are likely to have very "noisy" surroundings, whereas a driveway or path to a house is likely to be more "quiet" [3].

Local Binary Patterns (LBP) has been proved to be an effective texture descriptor in image processing and classification. The interest in LBP is due to its low computational complexity and high robustness to local variations [9]. It was proposed by T. Ojala et. al. [10] in 1996 and is calculated by comparing pixels with their neighbouring pixels. Divide the examined window into cells (e.g. 16x16 pixels for each cell). Based on this comparison a histogram is computed that can be used for training.

In previous experiments, feature sets based on individual pixels have been inefficient in detection of landing areas [3]. Hence, feature extraction in our system will be carried out for a patch of pixels. Patches will be labelled as appropriate for landing if it consists of the number of pixels suitable for landing is greater than a threshold value that is determined empirically. The exact numbers that will be used for our 
features, such as patch sizes, number of texture features, etc. will also be determined practically.

As we are implementing supervised learning, the data will be labelled through an image editor. A copy of the image will be edited so that all pixels in landing areas have the same colour, one that doesn't appear naturally in satellite images of houses. The feature vectors will then be calculated from the edited image.

\subsection{Training}

In this paper, we use Support Vector Machine (SVM) with three different kernels, namely linear kernel, radial basis function kernel (RBF) and the polynomial kernel.

SVM determines the hyper-plane that separates two classes of data and performs binary classification. Non-linear classification can also be performed using different kernels. We have selected SVM as in most cases it outperform other well-known classification methods, such as k-means [11].

\subsection{Roof Detection}

Roof detection is widely used for the detection of buildings, houses and residential areas [2] [12] [13]. Methods based on the colour of roofs, presence of shadows, or shape detection have been used in the detection of roofs and houses. In our system, we will use a colour based system that is used in [13]. Here images in RGB format will be used to extract the colour invariant properties. Multispectral information is obtained from satellite images as the red and near-infrared bands.

A colour invariant is defined with the red and green bands of the aerial image as

$$
\psi_{\mathrm{r}}=\frac{4}{\pi} \arctan \left(\frac{R-G}{R+G}\right)
$$

Here R and G stand for the red and green bands respectively. This colour invariant has value unity for red coloured objects and a value of minus unity for green objects. This colour invariant is independent of intensity. Therefore, the red rooftops of houses or buildings can be easily detected using $\psi \mathrm{r}$.

\subsection{Final Result}

Once the landing areas are identified by the training algorithm and roofs have been eliminated, we have to check if the landing area size is greater than the size of the parcel or UAV. This filtering process will remove any 'safe' landing patches that are smaller than a pre-set value of width $X$ height, since a safe landing site needs to be larger than this specified size. The result obtained after this filtering, will be the final image that highlights the areas that are appropriate for landing.

\section{CONCLUSION AND FUTURE SCOPE}

In this paper we have proposed a system to detect suitable landing areas when the delivery address is known. The process involves a few key steps, which can be summarized as collecting the satellite image based on a given address, extract colour and texture features through the use of RGB and HSV colour models, and edge detection algorithms. AN important part of the feature extraction process is roof detection which is achieved based on shape detection, as well as the presence of shadows. This is also achieved through the RGB colour model. We then use SVM algorithms for training of the system, for automated classification. Finally a safe area large enough is obtained, where the unmanned air vehicle or drone can possibly land. We are in the process of implementing the above method using MATLAB and hope to accomplish a high level of accuracy and consistency in our results. Future scope in this domain would be to develop a software that detects landing areas using known addresses and has a safety mechanism for landing in case of emergencies, i.e. forced landing. The project has some promising areas of application such as automated delivery systems using drones that can be used by online market giants, to make quicker and more efficient deliveries based on just an address of the recipient's location, reducing the amount of required man-power in the process. Some research has already been done in this domain ( [4]), and efforts should be made to collaborate both these types of systems.

\section{REFERENCES}

[1] E. F. A. M. P. Z. S. L. A. Cesetti, "A Vision-Based Guidance System for UAV Navigation and Safe Landing using Natural Landmarks," Springer Science + Business Media, 2009.

[2] D. Seyfallah Bouraoui, "A System to Detect Residential Area in Multispectral Satellite Images," IEEE, 2010.

[3] G. A. Josh Beal, "Detection of Landable Areas for Aerial Vehicles".

[4] S. D. C. F. L. M. S. S. Xufeng Guo, "Automatic UAV Forced Landing Site Detection using Machine Learning," in 2014 International Conference on Digital lmage Computing: Techniques and Applications (DlCTA), IEEE., 2014.

[5] R. D. D. H. J.W. Rouse, "Monitoring the vernal advancement of natural vegetation, Final Report," NASA/GCSFC, Greenbelt, MD, 1974.

[6] Y. Q. S. G. X. Wen Chen, "Identifying Computer Graphics Using HSV Colour Model and Statistical Moments of Charecteristic Functions," IEEE Transactions, 2007.

[7] W. B. C. J. C. B. Michael W. Schwarz, "An experimental comparison of RGB, YIQ, LAB, HSV, and opponent color models," ACM Transactions on Graphics, 1987.

[8] K. S. I. D. Robert M. Haralick, "Textural Features for Image Classification," IEEE Transactions on Systems, Man and Cybernetics., 1973.

[9] M. P. T. M. T. Ojala, "Multiresolution gray-scale and rotation invariant texture classification with local binary patterns," IEEE Trans. Pattern Anal. Mach. Intell, 2002.

[10] M. P. D. H. T. Ojala, "A comparative study of texture measures with classification based on featured distributions," Pattern Recognition, Elsevier, 1996.

[11] B.-S. K. S. S. Jinho Kim, "Comparing Image Classification Methods: K-Nearest-Neighbor and Support-Vector-Machines," Applied Mathematics in Electrical and Computer Engineering, 2012.

[12] D. W. Z. Sonke Muller, "Robust Building Detection in Aerial Images," Archives of Photogrammetry and Remote Sensing, 2005.

[13] C. U. Beril Sirmacek, "Building Detection from Aerial Images using Invariant Color Features and Shadow Information," IEEE Transactions, 2008. 